\title{
Usage Analysis of Smartphone with Hierarchical Clustering
}

\author{
R.Jayakarthik, M.S.Nidhya, K.Parvathavarthini
}

\begin{abstract}
Functional and Non Functional attributes are the important factors of the Smartphone. This paper deals with how to group the characteristics of mobile phone using the clustering algorithms and then obtained the results after classify the related algorithms. Classify all the functional and non-functional attributes of the smartphone using the latest data mining algorithms using WEKA (Waikato Environment for Knowledge Analysis) open-source software to analyze data.
\end{abstract}

Index Terms: Usage Survey, Hierarchical Clustering. WEKA tool.

\section{INTRODUCTION}

Innovative and advanced technology made the usage of Smartphone and its applications present in everything and everywhere. Smart Phone has various functional and nonfunctional attributes. Connectivity, video chatting, battery life, voice communication, data and some other common applications. There are several functional attributes like a long-standing battery, high-speed processing, luculent display, a great camera, numerous windows, high volume of memory, Infrared remote control. The number of nonfunctional attributes like simplicity, portability, recoverability, manageability, serviceability, scalability, modularity, customizability, security, variability, volatility, traceability, adaptability, usability, clarity, ubiquity, integrity, reconfigurability, etc.

\section{EXISTING APPROACH}

A recent study shows that [1] The usage of the smartphone by youth has been a global fact in recent years. It is now an integral part of young one's daily lives and used it's in the popular form of electronic communication. Entertainment, like online video gaming, listening to music and taking pictures and social networking also plays a vital role in a smartphone among students. Nevertheless, in spite of such handy and interesting features, it was found that 56 percent of female users were more engaged in mobile usage which ranged between super to addict stage while 11 percent had higher chances to get addicted than males. When survey was conducted on student class users by the University of Perpetual Help System, it was found that the results bordered between super to addict stage.

Revised Manuscript Received on October 12, 2019

Dr.R.Jayakarthik, Associate Prof,Department of Computer Science, VELS Institute of Science, Technology and Advanced Studies (VISTAS), Chennai, India.

M.S. Nidhya, Asst Prof, Department of Computer Science, VELS Institute of Science, Technology and Advanced Studies (VISTAS), Chennai, India.

K.Parvathavarthini, Asst Prof, Department of Computer Science, VELS Institute of Science, Technology and Advanced Studies (VISTAS), Chennai, India.
It was observed that the students launched the applications to which they were addicted 60 times within 24 hours, among which 12 percent were males while 23 percent were females. If the applications were launched between 16 to 60 times in a day, they fell under Super Users category among which 16 percent were males and 33 percent were females. Only 9 percent males and 6 percent females launched the applications for less than 16 times within 24 hours of the day

Usage of smart phones by college students: Though college students were found to be ranging between addicts to heavy users, it was found that they mostly visited sites that were on topics like illustrating work and presentations, using of dictionary applications, e-books/ articles/ news, debating topics on current happenings etc. All these fall under the category of learning and hence considered to beneficial for the betterment of knowledge among students based on the 5 parameters which were proposed by Lenhart and Maguth as it was calculated for mean value and the result was 3.13. Students mainly u se mobile for the sake of socializing, aid to learning, entertainment, emergency and for information in various topics which are easily available on web today, without any need of asking anyone. Students from undergraduate degrees mainly used mobiles for reflecting personal identity, communication on social websites, and entertainment. This research was mainly influenced by McQuail's typology to find out how important the usage of smart phones was. According to the results obtained, it was identified that most of the smartphone users confirmed that it was significant to have their personal identity. As per the results, it was evident that undergraduates especially from Malaysia consider their smartphones mainly for status sign, personal identity and self-respect for their personal lifestyle. Hence, it is evident to conclude that there is a drastic increase in the usage of smartphones and its popularity is increasing day by day. It appears that though the young ones have a conventional mobile phone, they still urge for smartphones as a significant and minimum need in order to serve their several needs in practicality. It is not surprising to know that they even consider having 2 phones in order to avoid low battery issues. Though the research tries to show that smartphone usage is a minimum need in present days, it clearly misses some basic points of intimate knowledge as the researcher failed to understand the feelings, insights and human viewpoints. As per the results found when data mining was applied to the 2,60,000 entries of log data which mainly recorded showing how 25 users accessed widespread apps on 26 devices while at work and daily lives during the period September 2010 till March 2011, the main focus was on data preparation. In order to obtain much more knowledge of the usage of apps, we have illustrated how to

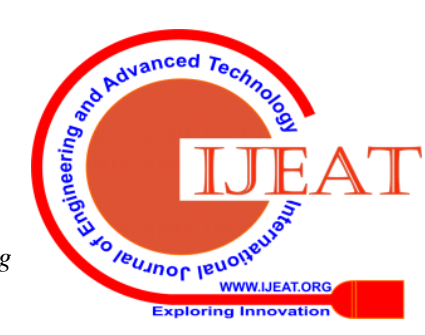


prepare data in order to apply to CARM Algorithm (Classic Association Rule Mining). Later, we also explained the regular sequential pattern mining algorithm so that they can know how to mine log data of smartphone apps usage. We have also discussed the rules and patterns generated in the results in order to identify with better knowledge for application developers, smartphone designers and researchers. It was evident that there were whopping number of users for WhatsApp application throughout the world and it was used for the highest amount of time when compared with other applications. This data obviously contributed to the better understanding of the importance of this communication channel. According to [5] revealed the trends in smartphone usage among students from highly developed metropolitan twin cities like Secunderabad and Hyderabad. A study has shown that most of the students use the smartphone in the college for making a phone call, practical video recording, using an online dictionary, taking pictures of class notes, sending a text message and studying. At home, participants use to browsing, listening to music, download software, playing online games and taking video, shopping cart, booking tickets. The least performed activity was to take pictures. In other areas, the users used smartphones for security (especially female participants), checking e-mails, taking video, listening to music, and watching videos and surfing net for different reasons like Language Translator, GPS for Location Navigation. The score rate for performing voice calls stood in the last position. A high percentage of learners used smartphone for educational purpose to take a snapshot of class notes or download the class notes. Most of the students can be able to own smartphone priced between 5,000 and 15,000. The majority of the students preferred Apple and Samsung followed by HTC, Blackberry, and others. Operating systems like Android and Windows were mostly preferred by many learners. According to [6], Web Usage Mining method is discussed by implementing the pattern discovery system using the association rules. On the pre-processed dataset, clustering approaches were implemented in order to group various transactions that were closely correlated. Examining such type of clusters will result in the discovery of robust association procedures. While working on large database transactions, we have achieved all the important association rules. We could identify the link between various webpage/ app requests. We have mainly considered the supporting and promising values obtained from rules in order to identify the interest of web visitors. Hence, the hit count was high when examining the attitude of the web visitor. The results obtained in this paper through this approach will be ery helpful to the web designers when they want to improvise the usability of their website by identifying the closely interrelated connections in the website. As per [7], the recommendation system called as 'Mobile Coupon', was applied. Depending upon consumer usage patterns, this method had produced various coupons for the users that use collaborative filtering system and association research method. Hence, the issue of information overhead was resolved. In order to improve convenience for users, the used information was collected by making use of implicit approach from NFC. While doing this, parallel work was conducted in selecting various business types. This increased the likelihood of users to make use of time slot through association method. In addition to this, determining purchase data of similar users for different business was identified through collaborative filtering system. This was mainly implemented to produce a recommendation list that focused around businesses which most of the users were unaware of or have not even tried even though they knew about it. Therefore, through this approach, both businesses and customers can make use of recommendation lists which were closely related to their requirement. As per [8], better understanding was achieved on analyzing frequent connecting websites and routine works through devices like smartphones and other devices that were a kind of throughout $\mathrm{ON}$ and stay connected. This paper clearly mentions how to determine the method of gathering sensor data through a smartphone that is inevitably handled, like, (i) the place of interest by a smartphone user along with their semantic label like work, home and more, (ii) the forecasted transitions among such places where we have tested our system on a group of student in order to achieve best results. Our approach accurately identified their movements and places of interest. Thus, the work in this paper clearly represented the opportunities for extension. In addition to this, when making use of huge information in order to retrieve staying mechanisms, you can precisely improve the activity of interference system. Also, on the parallel level, we shall act on methods for the usage of inferred data for tailored and situational recommendations. For instance, if you want the user to be presented with suitable data when at home, you may connect the preferences of browsing and locations respectively.

As per [10], in spite of apps market offering various restaurant recommender systems, there still exists the encountering of some of the innovative and research required challenges. In order to overcome some of the prevalent loopholes, this paper suggests the (i) the presenting of both group and individual interest in restaurant recommendation on the basis of like approach with similar application, and (ii) integration of data through various familiar and mostly used dining apps into a solo depository. To achieve centralized access of data, we need to bind the scattered data into one place, thus leading to the result of better resolution constructing support. In addition to this, a weighing system is provided to the users so that they can allocate more significance to their available platforms which gained their trust. So, this way, integrated data is considered for recommendation by taking count of the preferences made by solo users. These approaches were operated by making use of a mobile restaurant recommendation system, called as BomApetite, that groups various data and scores from restaurants through apps like Google places, Yelp, Foursquare, Zomato and last but not the least, Trip Advisor. A research was performed on users to calculate the amount of practicality and usability of the mobile recommendation system. The participants of the research conveyed that they were comfortable when data that they often use, was grouped from various platforms. The data access for grouped data happens through a single query omitting the need for making repeated searches of similar items in various apps. This helps to skip the need to compare afterward result comparison. Hence, this approach along with the succeeding decision approach is highly accurate and better informed. The user research provided yet another outcome was that, with a well-

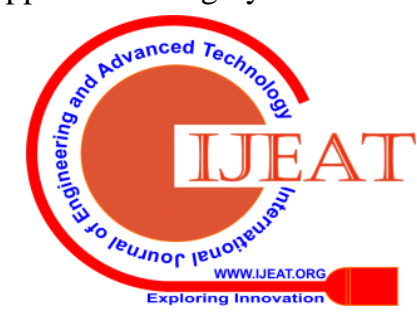


defined price duration, and more significantly, when a restaurant was selected through dining app was based on global restaurant level of rating, the trust was improved on the rating. The research clearly conveyed that the voting method and integration recommendation system was most convenient and practical for users. They agreed that it was innovative thought and many of them opined that they would continue with these features.

\section{METHODOLOGY}

My projected method was analysis the non-functional as well as functional quality attributes of the mobile phone depending upon the user experiences. Hence in the first step, we follow to predict the user experiences of the mobile phone. Then apply different data mining techniques for investigation.

We prepared the following survey to predict the user experiences about the mobile phone.

\author{
- $\quad$ Your Sex: Female, Male \\ - Your Age: 17 or Younger, 18 to 20, 21 to 29, 30 to \\ 39, 40 to 49, 50 to 59 and Above 60 .
}

- Your Education Level: School, Undergraduate, Postgraduate, PHD

- $\quad$ Spend on a mobile phone: Less than 5000, 5000 to 10000, 10000 to 15000,15000 to 20000, Above 20000

- Function, Quality in Service, Advertisement Friend's recommendation, Appearance, Brand image and last but not least Price are the Important factors of mobile phone

- Usage duration is less than 6 or 7 months to 1 year or more than 1 year.

- Causes to change your current mobile phone: Stolen or Broken, Lack of function, Out of fashion

- $\quad$ First mobile phone: iOS's Apple, Samsung, Sony, Nokia, Motorola, LG, Blackberry, HTC.

- Current mobile phone: Blackberry, Samsung, Motorola, Nokia, LG, HTC, Apple, Sony.

- Your favorite brand: Samsung, Apple, Sony, Nokia, Motorola, LG, Blackberry, HTC.

- Three features of a mobile phone: Video/ Cam Capabilities, Brand, Design, Technical Specifications (RAM, processor, Storage Space, etc.), Availability of applications, Product Dimensions/ Screen Size, Battery life, Ease of use, Operating System.

- The primary usage of your phone: Business, Social, Gaming

- Satisfactory level of the overall design of your mobile phone: Very Satisfactory, Most Satisfactory, Satisfactory, Not Satisfactory

- Does your mobile phone play out the administration directly at the first run through?

- Does your mobile phone provide services at the promised time? Often, Rarely, Sometimes, Occasionally

- Your mobile can adapt to the new functionality, services, and features easily: Often, Rarely, Sometimes, Occasionally

- Your mobile system can work accurately for different load conditions: Often, Rarely, Sometimes, Occasionally

- Your mobile can exchange the information internally and externally
Very Satisfactory, Most Satisfactory, Satisfactory, Not Satisfactory

- $\quad$ Your mobile can easily handle all the applications:

Very Satisfactory, Most Satisfactory, Satisfactory, Not Satisfactory

- Your mobile system can respond within a given time intervals: Often, Rarely, Sometimes, Occasionally

- Your mobile battery can remain operates over time: less than 3 hours, 3 to 5 hours, 5 to 8 hours, 8 to 12 hours, greater than 12 hours.

- Your mobile has provided secure apps

- Your mobile can simply identify and resolve issues: Very Satisfactory, Most Satisfactory, Satisfactory, Not Satisfactory

- Your mobile can give good user experience: Often, Rarely, Sometimes, Occasionally

Overall above 180 persons answered the questions. I was created .csv file using with the above data and it was shown different results as follows. The Male 90 and Female 90 persons have answered the questionnaires. The age group 18- 20 of the respondents were around 60 . The age bunch 21-29 of the respondents are around 60. The age group 3039 of the respondents are around 60 . Thus we concentrate on from 18 to 39 aged people are targeted because users of this age group people can analyze and answer the various qualities of the mobile phone. The respondent's educational qualification was 90 peoples finished their undergraduates. The respondent's educational qualification was 90 peoples finished their postgraduates. The $30 \%$ of the people spent $5000-10000$. The $60 \%$ of the people spent $10000-15000$.he $40 \%$ of the people spent $15000-20000$. The very important factor of the mobile phone which was consider while the user buying the mobile is quality. The second very important factor is the function. The somewhat important factor is Brand image and appearance. The neutral factor is advertisement and recommendation by friends. The user uses a maximum time period of the mobile phone is less than 1 year. The user uses a minimum time period of the mobile phone is 7 months to 1 year. The main reason why they are changing the mobile device is stolen or broken, lack of functions and then out of fashion.

Many of them told Nokia as my first mobile and some of them told Samsung as my first mobile an very few of them told Honor, Mi, Vivo, Appo as my first mobile phone. Regarding the current mobile phone, many of them told Samsung and apple is my current mobile phone. Many of them told apple as my favorite brand and some of them told Samsung as my favorite brand and very few them told Nokia as my favorite brand. The users consider Technical specifications and battery life are as features while they are buying the mobile phone. The user's primary usage is always social activity, gaming is a pass time and using for business is a rare activity.

An open source data mining tool Weka's (Waikato Environment for Knowledge Analysis) package is crated in weka's data files format ARFF (Attribute Relation File Format). It not only support data mining algorithms but it also supports data preparation and meta learners. A whole range of data preparation, feature selection and data mining

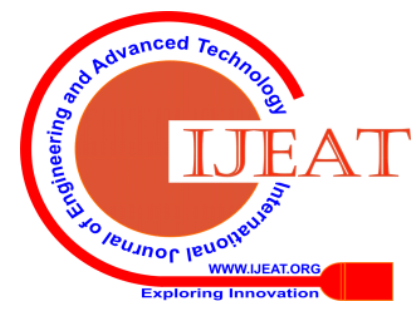


algorithms are integrated and needs several details of one and only one data format. Thus, makes trying and comparing different approaches really easy. It's a special attractive advantage of Weka.

We applied several steps in the dataset
A. Pre process
B. Apply cvparameterselection
C. Apply hierarchical clusters
D. Visualization

\section{A. Pre process}

Data pre-processing is the main crucial step. We need to clean data to produce good results. This process is used to identify the smooth out noisy data and outliers, correct inconsistent data and smooth the noisy data.

\section{B. CVParameterselection}

It is used to choose the best value for a parameter. On the It looks for the best parameter set by upgrading crossapproved exactness on the preparation information. Each worth is assessed utilizing ten times cross-approval.

Optimization of parameters is specified using the CVParameters field

For each parameter, three-section of information must be supplied:

(1) The quantity of steps to attempt in this range

(2) A numeric scope of qualities to assess; and

(3) A string that names it utilizing its letter code

Use CVParameterSelection related to IBk to choose the best an incentive for the local size, extending from 1 to 10 out of 10 stages. The letter code for the local size is K. The crossapproved precision of the parameter-tuned adaptation of IBK is legitimately tantamount with its exactness utilizing default settings since tuning is performed by applying internal cross-approval races to find the best parameter esteem for each preparation set happening in the external crossapproval and the concluding yields the final performance estimate.

\section{Estimate Error Rates}

Split the informational index into $\mathrm{k}$ subsamples, use $\mathrm{k}$ 1 subsamples as preparing information and one subsample as test information fork overlap cross-approval

\section{Hierarchical Clustering}

It is a type of clustering algorithm method used to group the data objects into a tree of clusters. It can be classified as either agglomerative or divisive.

\section{Agglomerative Hierarchical Clustering}

This technique begins by putting each article in its very own bunch and after that unions these nuclear groups into a bigger and bigger bunch until the majority of the items are in a solitary bunch or until certain end conditions are fulfilled.

\section{Visualization of Data}

It gives the best results in most of the numeric data. The main GUI shows a histogram for the attribute distribution of a single selected attribute at a time and by default. Individual colors within a histogram column indicate individual classes.

\section{RESULTS AND DISCUSSION}

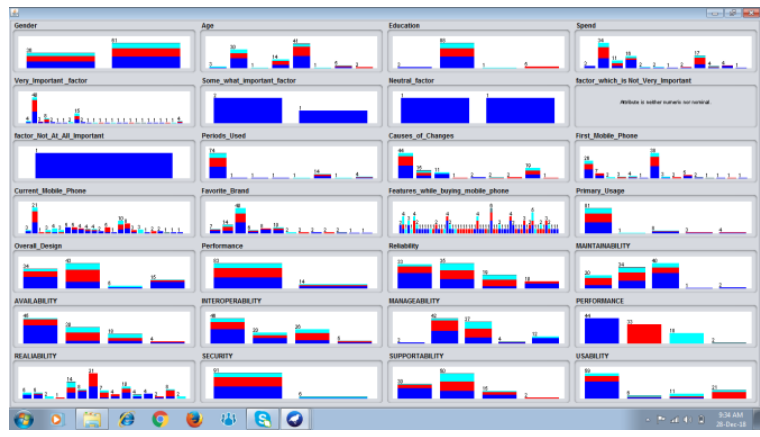

Fig. 1.Graphical attributes presentations of Smartphone Data

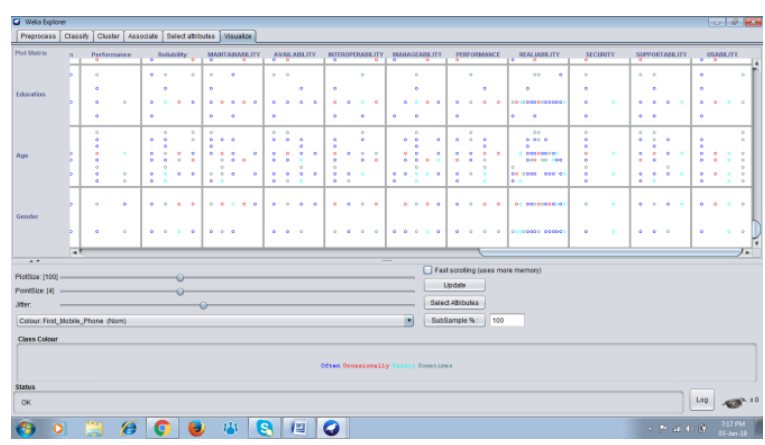

Fig. 2.Scatter Plots of the Smartphone Data

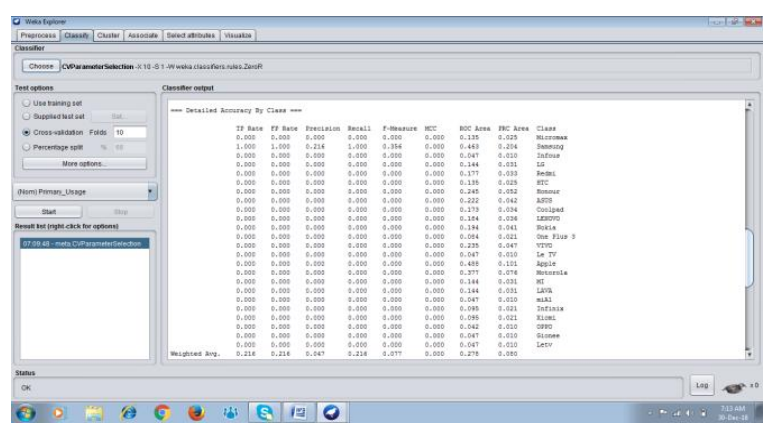

Fig. 3.CVParameterSelection Classifier for Primary Usage Data 


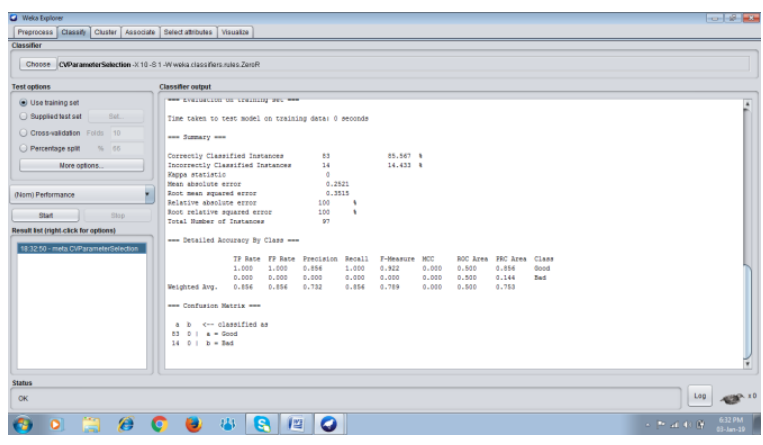

Fig. 4.CVParameterSelection Classifier for Performance Data

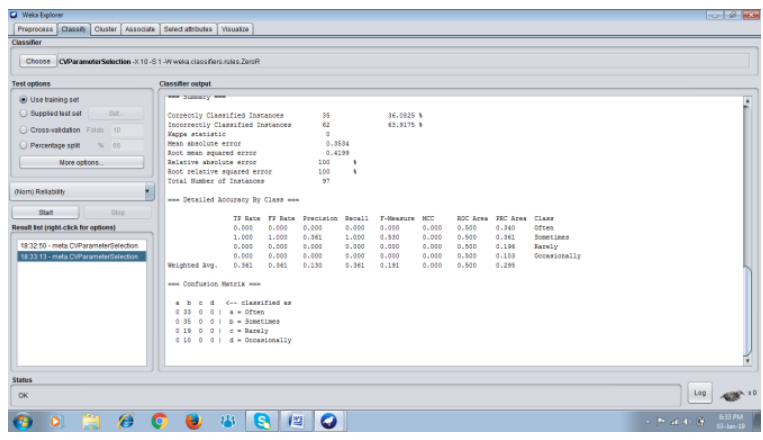

Fig. 5.CVParameterSelection Classifier for Reliability Data

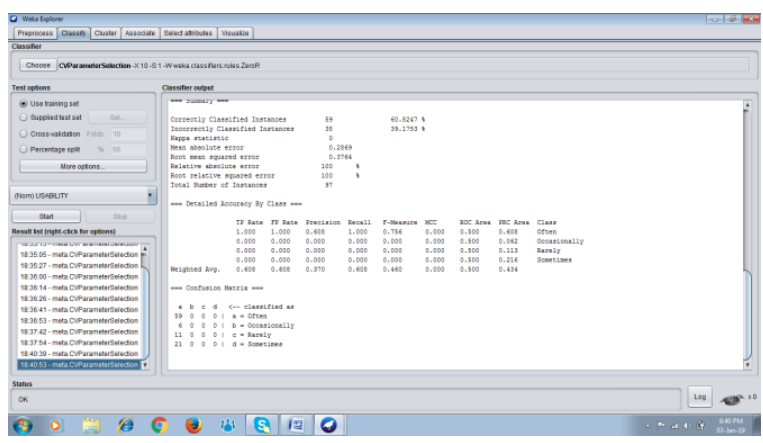

Fig. 6.Hierarchical Cluster for Usability Data

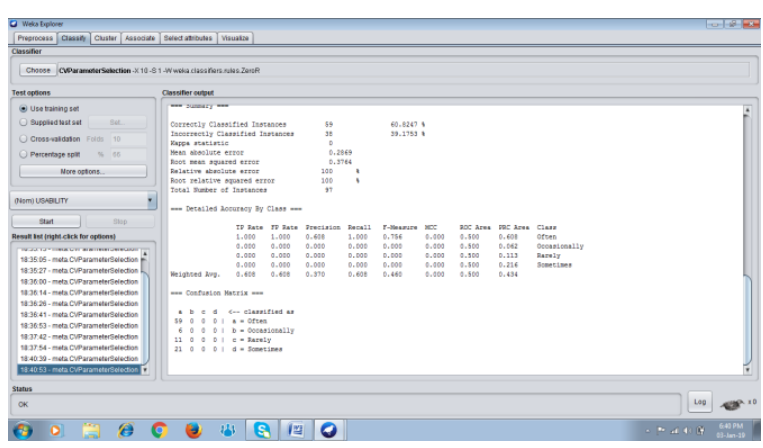

Fig. 7.Hierarchical Cluster for Performance Data

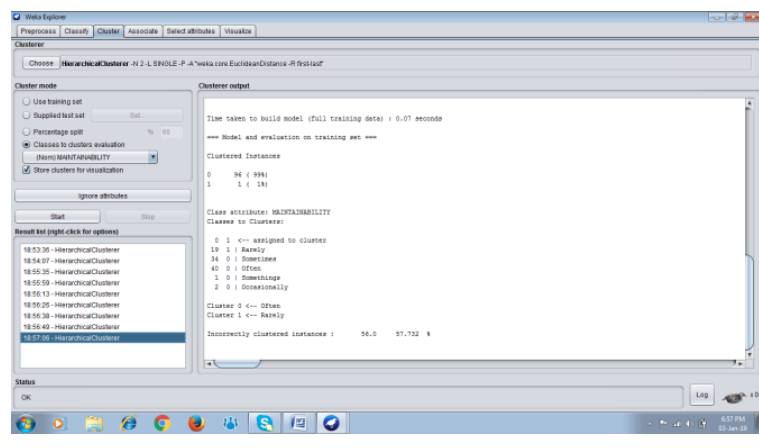

Fig. 8.Hierarchical Cluster for Maintainability Data

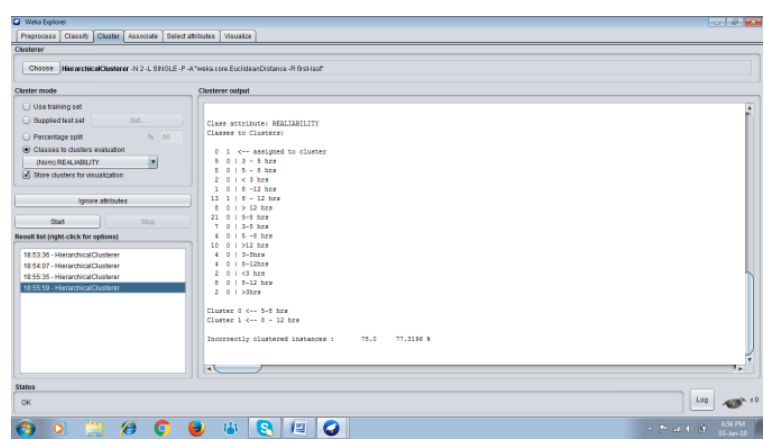

Fig. 9.Hierarchical Cluster for Reliability Data

\section{CONCLUSION}

The present method analyzed the user view about the functional and non-functional attributes of the Smartphone. Then all the views are applied in the different data mining techniques like Preprocess, Clustering, and classification. These results are obtained using weka in a structured format and these results show potential.

\section{REFERENCES}

1. Borst, S., "User-level performance of channel-aware

2. scheduling algorithms in wireless data networks", IEEE INFOCOM, 2003.

3. Balazinska, M., and Castro, P., "CharacterizingMobility and Network Usage in a Corporate Wireless

Local-Area Network," ACM MobiSys, May 2003. Bai, F., and Helmy, A., "A Survey of Mobility Modeling and Analysis in Wireless Adhoc Networks",

4. Book chapter, Springer, Oct 06, ISBN: 978-0-387-25483-8.

5. Woan-Rou Tseng and Kuo-Wei Hsu, "Smartphone App Usage Log Mining ", International Journal of Computer and Electrical Engineering, Vol. 6, No. 2, April 2014.

6. Bai, F., Sadagopan, N., and Helmy, A., "The IMPORTANT Framework for Analyzing the Impact of Mobility on Performance of Routing for Ad Hoc Networks", AdHoc Networks Journal Elsevier, Vol. 1, Issue 4, pp. 383 -- 403, Nov. 2003.

7. Tang, D., and Baker, M., "Analysis of a Metropolitan-Area Wireless Network," Wireless Networks, vol. 8, no. 2-3, pp. 107 -120, Nov. 2004.

8. Kotz, D., Essien, K., "Analysis of a Campus-wide Wireless Network," ACM MobiCom, September, 2002. Christian Montag1,3*, Konrad Błaszkiewicz2, Rayna Sariyska1, Bernd Lachmann1, Ionut Andone2, Boris Trendafilov2, Mark Eibes2 and Alexander Markowetz2, "Smartphone usage in the 21st century: who is active on WhatsApp?". Montag et al. BMC Res Notes (2015) 8:331 DOI 10.1186/s13104-015-1280-z.

9. $\quad$ S.Saraswathi "Smartphone Usage Among Students", E-ISSN No: 2454-9916 | Volume : 3 | Issue: 6 | June 2017.

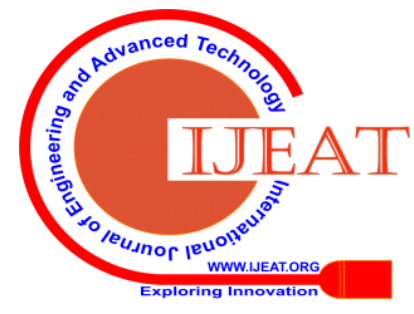


10. Ms. Kiruthika M, Mr. Rahul Jadhav, Ms. Dipa Dixit, Ms. Rashmi J, Ms. Anjali Nehete, Ms. Trupti Khodkar, "Pattern Discovery Using Association Rules" (IJACSA) International Journal of Advanced Computer Science and Applications, Vol. 2, No. 12, 2011.

11. JinHyun Jooa, SangWon Bangb, GeunDuk Parka, "Implementation of a Recommendation System using Association Rules and Collaborative Filtering" 1877-0509 @ 2016 Published by Elsevier B.V. This is an open-access article under the CC BYNC-ND license http://creativecommons.org/licenses/by-ncnd/4.0/). Peer-review under responsibility of the Organizing Committee of ITQM 2016 DOI: 10.1016/j.procs.2016.07.115.

12. G. Maggiore, C. Santos, A. Plaat, Smarter smartphones: understanding and predicting user habits from GPS sensor data, $1877-0509 @ 2014$ Elsevier B.V. This is an open-access article under the CC BY-NC-ND license (http://creativecommons.org/licenses/by-nc-nd/3.0/). Selection and peer-review under responsibility of Conference Program Chairs DOI: 10.1016/j.procs.2014.07.027.

13. Data mining with WEKA, Part 1 Introduction and regression Michael Abernethy Published on April 27, 2010. https://www.ibm.com/developerworks/library/os-weka1/

14. Gabriel Marquesa, Ana Respícioa,b*, Ana Paula Afonso, A mobile recommendation system supporting group collaborative decision making, 1877-0509 @ 2016 The Authors. Published by Elsevier B.V. This is an open-access article under the CC BY-NC-ND license (http://creativecommons.org/licenses/by-nc-nd/4.0/). Peerreview under responsibility of KES International DOI: 10.1016/j.procs.2016.08.235

\section{AUTHORS PROFILE}

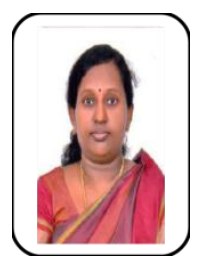

Dr.R.Jayakarthik, got her doctorate from Madurai Kamarajar University, Madurai, Master certificate in Information Technology and Master of Philosophy in the Computer Science from Madurai Kamarajar University, Madurai. She is as of now filling in as an Associate Professor in Department of Computer Science, Vels Institute of Science, Technology and Advanced Studies (VISTAS), Chennai.

She is having 10 years of instructing background. She has numerous productions in presumed diaries, for example, IEEE and Scopus. She has likewise enlisted and distributed Patents. She got Best Scientist Award in Global Education and Corporate Leadership Awards 2018. Her exploration intrigue incorporates Web Engineering, Cloud Computing, and Data mining. She distributed multiple Books. She conveyed different visitor teachers in Web Engineering, Software building and so on. in Web Engineering, Software engineering etc.

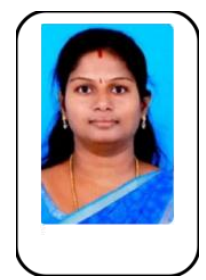

Dr.M.S.Nidhya , earned her doctorate from Bharathiar University, Coimbatore, Master certificate in Computer science and Master of Philosophy in a similar field from Bharathidasan University, Trichy. She is qualified with SET.She is at present filling in as an Assistant Professor in Department of Computer Science, Vels Institute of Science, Technology and Advanced Studies (VISTAS), Chennai.

She is having 13 years of educating knowledge. She has numerous distributions in rumored diaries, for example, IEEE and Springer. She has additionally enrolled anddistributed Patents. She got Best Academician Award in Global Education and Corporate Leadership Awards 2018. Her exploration intrigue incorporates Wireless Sensor Networks, Cloud 